\title{
Análisis constructivo de la cúpula de madera del desaparecido Palacio de los Cárdenas en Torrijos (S. XV)
}

\section{Constructive analysis of the wooden dome in the disappeared Cárdenas Palace in Torrijos $\left(X V^{\text {th }} C\right.$.)}

\author{
Enrique Nuere Matauco ${ }^{(*)}$, Ángel Candelas-Gutierrez ${ }^{(*)}$, Javier de Mingo García ${ }^{(* * *)}$
}

\section{RESUMEN}

En el Museo Arqueológico Nacional se halla una de las cuatro techumbres de lazo que existieron en el desaparecido y expoliado Palacio de los Cárdenas en Torrijos (Toledo, s. XV), de las cuales era la única en forma de media naranja. La excepcionalidad de las cúpulas en la carpintería de armar española hace de cada una de ellas un ejemplar único. Saber trazarlas permitía alcanzar el más alto grado del gremio. Sin embargo, en los tratados no llega a completarse una correcta definición de los procedimientos para su trazado, construcción e integración de laceria. Tras el análisis in situ de esta armadura desarrollamos tanto su descripción formal como su configuración constructiva; desvelamos cual es su estructura resistente y avanzamos en los procedimientos que se pudieron seguir tanto para la introducción del lazo en la dificil geometria esférica, como para llevar a cabo su fabricación y montaje.

Palabras clave: Cúpula, carpintería tradicional, tratados de arquitectura, historia de la construcción, trazados de lazo, madera, Toledo.

\section{ABSTRACT}

In the National Archaeological Museum there is one of the four wooden ceilings with interlaced joinery that existed in the towers of the disappeared and plundered Cárdenas Palace in Torrijos (Toledo, $X V^{\text {th }} C$.), that was the only one with dome form. The exceptionality of this kind of domes in the Spanish joinery made each one of them a unique example. Knowing how to draw them allowed to reach the highest degree of the guild. However, in the treatises, a correct definition of the procedures for its layout, construction and integration of the interlaced pattern is not completed. After the in situ analysis of this timber frame we develop both its formal description and its constructive configuration; we reveal what is its resistant structure and we advance in the procedures that could be followed both for the introduction of the interlaced pattern in the difficult spherical geometry, and for carrying out its manufacture and assembly.

Keywords: Dome, historic timber frames, architectural treatises, construction history, interlacing patterns, timber, Toledo.

(*) Dr. Arquitecto. Real Academia de Bellas Artes de San Fernando, Madrid (España).

(**) Dr. Arquitecto. Universidad de Sevilla (España).

(***) Arquitecto. Universidad Politécnica de Madrid (España).

Persona de contacto/Corresponding author: alcg@us.es (Ángel Candelas-Gutierrez)

ORCID: http://orcid.org/oooo-0002-6368-9402 (E. Nuere); http://orcid.org/oooo-0oo2-7599-4498 (A. Candelas-Gutierrez); https://orcid.org/oooo-0003-3829-6081 (J. de Mingo)

Cómo citar este artículo/Citation: Nuere Matauco, Enrique; Candelas-Gutierrez, Ángel; de Mingo García, Javier (2020). Análisis constructivo de la cúpula de madera del desaparecido Palacio de los Cárdenas en Torrijos (S. XV). Informes de la Construcción, 72(559): e353. https://doi.org/10.3989/ic.71019.

Copyright: (C) 2020 CSIC. Este es un artículo de acceso abierto distribuido bajo los términos de la licencia de uso y distribución Creative Commons Reconocimiento 4.0 Internacional (CC BY 4.0). 


\section{INTRODUCCIÓN}

$\mathrm{Al}$ enfrentarnos al estudio de las medias naranjas, término usado desde hace más de cuatro siglos por los antiguos carpinteros de lo blanco para designar cúpulas de madera, nos asombra el dominio geométrico de quienes fueron capaces de diseñarlas. De ahí el enorme interés en llegar a saber qué proceso siguieron sus autores desde su concepción hasta su realización. En el presente trabajo hemos efectuado un acercamiento lo más realista posible al hipotético camino seguido por los artífices de dichas construcciones, desde el trazado hasta el montaje, intentando superar la escasez de información o incluso la ignorancia de los tratadistas que intentaron aportar por escrito algo sobre el tema.

\section{CÚPULAS DE MADERA EXISTENTES}

En la actualidad se tiene constancia de seis únicas cúpulas de madera de lacería que puedan encuadrarse dentro de carpintería de armar hispánica: la del Salón de Embajadores de los Reales Alcázares de Sevilla (h. 1427), la procedente del Palacio de los Cárdenas de Torrijos que aquí se estudia (s. XV), la de la Casa de Pilatos de Sevilla (s. XVI), la que se erigió sobre la escalera del Convento de San Francisco de Lima (s. XVII), actualmente reconstruida y, aunque con dimensiones muy reducidas y ataujeradas, las no menos importantes del Patio de los Leones de la Alhambra (s. XIV). Incluso sin tener en cuenta la labor de lazo, las cúpulas semiesféricas con estructura resistente vista de madera son una rareza de la cual existen escasos ejemplares en el mundo.

No obstante, sí existen diversas cúpulas de madera con estructura oculta, cuyos elementos no están sometidos a complejos condicionantes que aúnen cuestiones estructurales y visuales, tales como las cúpulas artesonadas del Hospital Real de Granada y de la iglesia de Copacabana en Potosí (Bolivia). También existen algunas cúpulas de madera producidas fuera del contexto estrictamente hispano, como la cúpula de la Roca de Jerusalén, la de la Gran Mezquita de Damasco o algunas de las documentadas por Pavón Maldonado (1). Más habituales incluso, pero ya mucho más tardías, fueron las cúpulas encamonadas, también de madera pero concebidas para ser revestidas interiormente de yeso, simulando ser de fábrica (2). Sin poder ser consideradas cúpulas, son mucho más relativamente frecuentes las bóvedas nervadas de madera que se pueden encontrar en diversos países europeos incluyendo el nuestro, sobre todo en País Vasco y Galicia.

En cualquier caso, ninguna de las mencionadas en el párrafo precedente se aproxima al método constructivo de las primeras, cuyos elementos resistentes quedan a la vista, y forman a su vez parte del acabado decorativo de la cúpula.

Por orden cronológico las primeras en aparecer, en el siglo XIV, fueron los cupulines ataujerados que hay a ambos extremos del Patio de los Leones de la Alhambra, si bien su técnica y trazado son completamente distintos al resto de las cúpulas que más tarde se realizarían en Castilla y América. Su forma completamente esférica, y su trazado compuesto a base de ruedas de once brazos no se volverían a repetir en ninguna otra techumbre de madera, a pesar de lo cual, se las podría considerar antecedentes del resto de las cúpulas que vinieron a continuación, las cuales, si bien carecían de una aplicación tan directa de la geometría esférica, constructivamente pasa- ron a ser apeinazadas. La depurada y perfecta técnica de trazado de los cupulines parece estar directamente relacionada con la trigonometría esférica, cuyo desarrollo fue muy importante en Al-Ándalus y también en diversas obras y traducciones de textos que se hicieron en la Escuela de Traductores de Toledo, como por ejemplo el Almagesto de Ptolomeo, o el teorema de Jabir Ibn Aflah.

Las ruedas de las cúpulas del Patio de los Leones tienen precisamente once brazos debido a la poligonación esférica de su trazado, que está compuesto a imitación del lazo plano de doce. Es decir, partiendo de una base plana en la que los ejes de las ruedas de doce tienen un ángulo de abertura de $30^{\circ}$, al aplicar la doble curvatura, y ser vigentes las leyes de la geometría esférica, se adapta el diseño hasta eliminar uno de sus brazos, aunque ello implique realizar aproximaciones en algunos ángulos. La geometría de estas cúpulas fue estudiada con gran detalle por el ingeniero Prieto y Vives (3).

A partir del siglo XV se realizaron las cuatro cúpulas ya referidas anteriormente, que han sobrevivido hasta nuestros días, tres de ellas en España y una en Perú. Todas ellas usaron el trazado de lazo lefe, basado en la repetición seriada de ruedas de diez brazos, como sustrato compositivo de la ornamentación. La descripción más sintética de lazo lefe la da Nuere (4): "La labor de lazo de 10 con ruedas de azafates redondos exclusivamente, sin modificaciones en la traza. Los huecos existentes en el conjunto se rellenan con parejas de los mismos azafates". La secuencia constructiva que siguieron los tres ejemplares españoles partía de la realización de doce gajos en los cuales, los camones principales, colocados como meridianos en los límites del gajo, conformaban la estructura fundamental para llevar a cabo el trazado y la fabricación del resto del armazón, en cuanto a lacería se refiere.

La primera de ellas aparece en 1427, según González de León (5), en el Salón de Embajadores de los Reales Alcázares de Sevilla (6), llevada a cabo por el maestro D. Diego Ruiz. A finales del siglo XV se construye el Palacio de los Cárdenas en Torrijos, y en él, la cúpula que formaba la techumbre de la torre noreste, en la que se centra el presente estudio. Ya en 1537 se construye la media naranja sobre la escalera de la Casa de Pilatos (7), y finalmente, como última cúpula de las que se tiene noticia, en el Perú del siglo XVII, la que se ubica sobre las escaleras del Convento de San Francisco de Lima. Actualmente dicha cúpula es en realidad una réplica, realizada en 1973 tras su destrucción en 1940 por un terremoto y posiblemente con errores respecto a la original (8). Se diferencia de todas las anteriores en que está formada por ocho gajos en lugar de los doce habituales.

\section{CÚPULAS DE MADERA EN LOS TRATADOS}

Como muestra de la complejidad que encerraba la elaboración de la denominada media naranja, en las Ordenanzas de la Ciudad de Sevilla de 1527, en el folio CXLVIII (v) se recogen las pruebas que debían pasar los distintos oficios relacionados con la carpintería (vigoleros, de lo prieto, entalladores o carpinteros), de entre las cuales, la exigida para llegar al grado más alto, el de "Jumétrico" o "geométrico", era precisamente la de "saber fazer una quadra de media naranja de lazo lefe".

Por otra parte, las cúpulas de madera conforman un tema que aparece en dos de los tratados españoles más importantes en 
cuanto a carpintería de armar se refiere: el texto impreso de López de Arenas (9) y el manuscrito de Fray Andrés de San Miguel (10). Ambos describen en sus tratados tanto la media naranja o cúpula esférica, como la media caña o bóveda de rincón de claustro, si bien en ninguno de los casos se llega a abarcar por completo la totalidad del proceso constructivo.

\subsection{Diego López de Arenas}

López de Arenas escribió en sus manuscritos y tratados fundamentalmente recetas prácticas para otros carpinteros, soslayando lo que un profesional de dicho gremio conocía por lo común de su aplicación. En lo referente a cúpulas, dedicó sus esfuerzos a resaltar algunos aspectos de su realización, en los que sin embargo se perdía a la hora de desarrollarlos gráfica y textualmente, haciendo irreproducible su método en la realidad.

En su segundo manuscrito describe la forma de realizar una media naranja, dividiendo el plano en gajos que formarán, una vez curvados, la octava, décima o doceava parte de la misma (Figura 1). El problema estriba en que además de omitir la explicación de cómo dar la forma a los bordes de cada gajo, algo que posiblemente desconocía, las explicaciones que da están incompletas y equivocadas. A tenor de lo observado en sus obras, es probable que copiase dibujos de otros autores de los cuales desconocía su significado, puesto que algunos de ellos tienen propiedades singulares que no explica, y de los que sin embargo intenta extraer principios que no se cumplen.

En cuanto a los gajos, comienza describiendo la longitud de cada uno, que según explica, coincide con la línea KL, que subdivide en 11 partes al usar la proposición 32 del libro primero de Arquímedes, en el que se relaciona las longitudes de la circunferencia y su diámetro mediante la fracción 22/7, cuyo error respecto a $\pi$ es inferior al $0,04 \%$. No obstante, carece de todo sentido hacerlo a posteriori, cuando según el dibujo, la longitud KL viene determinada por la intersección con el bolsor, que es la continuación de la semiesfera bajo el plano ecuatorial, y no tiene en cuenta que la longitud del gajo incluye el propio bolsor.

Por otra parte, el dibujo que usa Arenas (Figura 1) cumple una propiedad que no explica: la longitud del segmento KL es idéntica a la del arco de círculo KM, y la justificación es simple: suponiendo que el radio de la circunferencia mi-

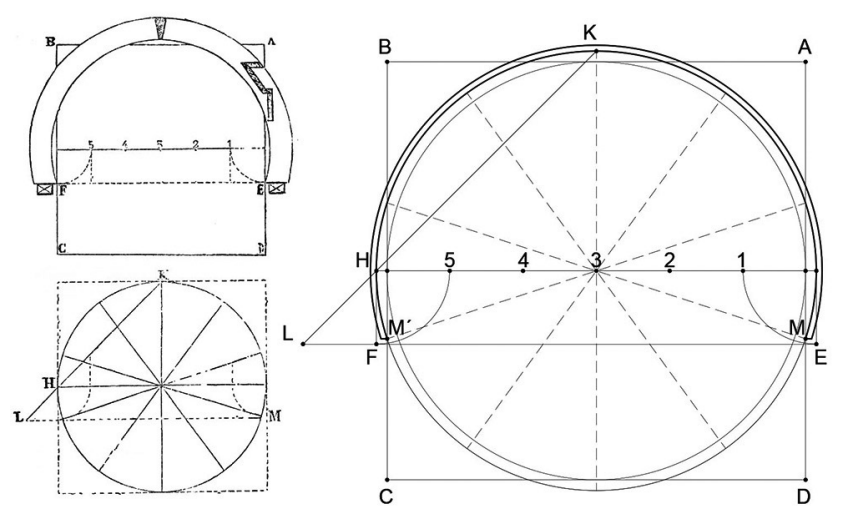

Figura 1. La media naranja en la lámina 33V del tratado de Arenas (izquierda), e interpretación de la misma en la investigación actual (derecha). diera tres unidades, al tratarse de un triángulo rectángulo isósceles, por el teorema de Pitágoras la hipotenusa HK medirá $3 \sqrt{ } 2$, y la correspondiente HL al triángulo externo a la circunferencia, cuyos catetos miden una unidad, medirá $\sqrt{ } \mathbf{2}$. Así pues, la longitud de $\mathrm{LK}$ es por tanto igual a $4 \sqrt{ } 2$. Por la otra parte, la longitud de un arco de meridiano de un gajo comprendido entre las intersecciones $\mathrm{K}$ y M, cuyo ángulo es $3 \pi / 5$, es $9 \pi / 5$. Si se comparan las dos longitudes, la diferencia entre ambas no llega ni al 2 por 1000, por lo que se puede considerar un cálculo con una aproximación bastante certera.

Ahora bien, olvidó Arenas que estaba usando como base para el cálculo, según su dibujo, el diámetro de un círculo que es la sección de la esfera a la altura del bolsor, y no el diámetro de la propia esfera, lo cual invalida la operación. Parece probable que Arenas estuviese usando alguna aproximación gráfica a la cuadratura del círculo basada en Arquímedes, y que al intentar adaptarla al caso concreto de una cúpula con bolsor, se confundiese. Aun así, basta tener en cuenta los errores cometidos para poder usar el dibujo correctamente, cosa que es muy probable que el autor del dibujo original sí supo hacer.

En la figura 2, López de Arenas indica cómo obtener los doce gajos de una media naranja, más lógico que hacerlo de diez, por la mayor facilidad de división. Cada gajo se divide en seis partes desde el ecuador al polo, y en cada punto de la división realizada se trazan unos radios, los cuales permitirán calcular la anchura del gajo a esa altura concreta.

Para mostrar un ejemplo, (Figura 2) se ha escogido el primer paralelo por encima del ecuador, empleando la forma gráfica que López de Arenas usa para calcular la longitud de la circunferencia. Forma un triángulo rectángulo cuyo cateto vertical es igual a tres veces el radio. Su hipotenusa tendrá un valor igual a la raíz cuadrada de tres al cuadrado más uno al cuadrado, es decir: de nuevo la raíz de diez. En el esquema hecho con el método de Arenas, la línea dibujada representa la longitud total del paralelo medido, por lo que la medida correspondiente a un gajo, a la altura del paralelo escogido, sería su doceava parte, es decir BC.

Por el mismo procedimiento obtendríamos el resto de distancias a los bordes del gajo, hoy es más sencillo (y más exacto) calcularlo numéricamente. El dibujo de Arenas que aparece en su libro impreso, donde explica la forma de dibu-
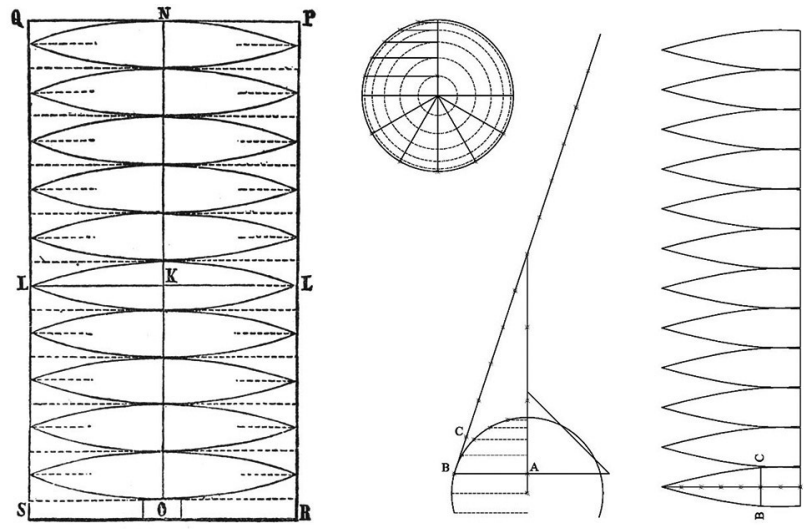

Figura 2. Subdivisión en diez gajos en la lámina 34R del tratado de Arenas (izquierda) y método propuesto de doce gajos (centro y derecha). 
jar los gajos, muestra varios errores: para empezar no se da cuenta que la circunferencia que ha calculado es la correspondiente al diámetro del bolsor que forma la media naranja, con lo que la longitud del gajo ya es errónea, aunque no la de la base de los gajos, pero no advierte que dichas bases son menores que el propio gajo en el ecuador, cuya mayor dimensión es la que corresponde al diámetro máximo. Por supuesto, no se para a considerar cuál es la forma de los bordes de cada gajo, sino que traza una curva de compas con un radio que escoge arbitrariamente, en vez de realizar el cálculo antes expuesto, relativamente simple, sobre todo si lo hacemos aritméticamente.

\subsection{Fray Andrés de San Miguel}

Fray Andrés de San Miguel se centra más en el aspecto teórico del trazado sobre el papel, sin entrar en los pormenores de lo constructivo a la hora de realizarlo en madera. Así, en la única referencia a la media naranja de su manuscrito, describe la manera de trazar el desarrollo plano de un huso de una semiesfera de un radio determinado sin explicar nada de cómo se introduce el lazo en ella.

\subsection{La interpretación posterior de los tratados}

Existen autores recientes que al interpretar los tratados, han incluido referencias a la media naranja, con análisis del asunto que varían en cuanto a su profundidad entre unos y otros. Por parte de los que se dedicaron a estudiar el tratado y los manuscritos de Arenas, fue Prieto y Vives el primero en analizar someramente lo que aquél contemplaba acerca de las cúpulas y dar cuenta de los errores y omisiones que cometió. Nuere (11) desentraña de manera definitiva los textos de Arenas, y es el único que propone el desarrollo de una media naranja, sorteando los errores del autor. Posteriormente, Toajas Roger (12), también refiere los fallos del sevillano, sin analizar la cuestión con más detalle. Por último, CandelasGutierrez (13) realiza importantes observaciones al trazado de cúpulas según Arenas, lo que le sirve para analizar más tarde el ejemplar de los Reales Alcázares de Sevilla con gran profundidad (14).

Por otro lado, en lo referente al manuscrito de Fray Andrés de San Miguel, Báez Macías (15) en realidad hace una transcripción de su manuscrito, sin analizar su contenido. Pero Nuere (16) vuelve a descodificar el mismo mediante el método de la lectura dibujada, aplicándolo en este caso al desarrollo del gajo que plantea San Miguel.

\section{LA CÚPULA DEL PALACIO CÁRDENAS}

\subsection{Introducción histórica}

Entre 1902 y 1904 se desmanteló el palacio que construyó en Torrijos (Toledo) a partir de 1482 Don Gutierre de Cárdenas, maestresala de Isabel la Católica. El palacio se refiere en diversas publicaciones con el nombre de los distintos títulos nobiliarios de los sucesores del promotor, así aparece denominado como Palacio Cárdenas, Palacio de Altamira o Palacio de Duques de Maqueda, el cual fue levantado sobre los restos del primitivo palacio de Pedro I.

Don Gutierre de Cárdenas fue una figura de excepcional influencia en el reinado de lsabel de Castilla y Fernando de Aragón, por lo que resulta verosímil admitir que cuando construyó su palacio en su tierra de origen habría podido implicar a algunos de los mejores artesanos de la época.

Poco antes de su demolición, Repullés y Vargas (17) publicó una descripción del palacio incorporando un plano de la planta baja del edificio (Figura 3), del que destaca la fachada, el patio, y fundamentalmente reseña con cierta profusión de detalles las armaduras que albergaban los cuatro cuartos de cada una de las esquinas del palacio, de manera que pueden ser identificadas en función de su forma y su decoración. De las cuatro salas destaca que "sus muros y armaduras de cubierta se elevan sobre los del resto del edificio, a modo de torreones", para posteriormente pasar a la descripción de cada una de ellas. Así, por ejemplo, refiere una "cúpula semiesférica cuajada de arabesca tracería" situada al noreste del edificio, una cubierta "de pabellón octogonal, decorada también con lazos y conchas" al otro lado del gran salón existente en su centro, un "doble artesonado octogonal, dividido en diez y seis compartimientos" al extremo de la crujía norte, y una "cúpula octogonal, que no tiene divisiones y está decorada con tracería geométrica” al extremo de la crujía sur.

Estas cuatro armaduras se ubican actual y respectivamente en la sala 26 del Museo Arqueológico Nacional (Madrid), en el Victoria and Albert Museum (Londres, Reino Unido), en el Legion of Honor Museum Of San Francisco (EEUU) y en una sala del Château de Villandry (Francia). Sin embargo, tuvieron otras ubicaciones intermedias tras el desmantelamiento del palacio que las albergaba. Perez Suescun (18) incorpora noticias de tales ubicaciones.

La dispersión de las techumbres fue el resultado de su venta por parte de los propietarios D. Enrique Villanueva Hilandera y D. Teófilo Díaz-Prieto, como ya se ha mencionado, en los primeros años del siglo XX. De ello da buena cuenta el artículo "El palacio de Torrijos" (19), que constituye prácticamente un catálogo de venta de las obras artísticas contenidas en el palacio, siendo muy elocuente el texto en el que incita a la aristocracia a no desperdiciar "esta ocasión que se les presenta para que por poco precio puedan ostentar en sus moradas joyas de tanto mérito".

Concretamente sobre la cúpula hoy existente en el Museo Arqueológico Nacional, Pérez Suescun indica que tras el desmantelamiento del palacio, "Don Platón Páramo, amante de las antigüedades, adquirió esta techumbre, la más rica, y construyó en Oropesa una especie de torre para albergarla.

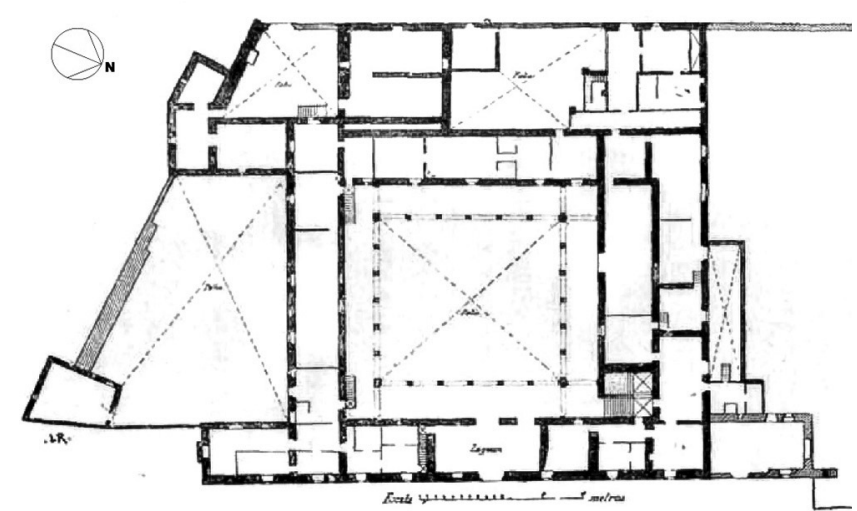

Figura 3. Planta baja del Palacio de los Cárdenas. Fuente: El palacio de Torrijos. Resumen de Arquitectura, p. 104. 
El Museo Arqueológico Nacional la adquirió a sus herederos en 1969". La torre en cuestión fue levantada ex-profeso para contener la cúpula en el antiguo museo de Cerámica de Oropesa, creado por D. Antonio, y estaba ornamentada exteriormente en un estilo a caballo entre el neomudéjar y el neogótico. Fue su hija Adela Páramo, la primera administradora del Parador de Oropesa, quien vendió la techumbre al Museo Arqueológico Nacional, motivo por el cual tal vez algunas fuentes indican erróneamente que estaba ubicada en dicho parador.

\subsection{Descripción de la cúpula del Museo Arqueológico Nacional}

La cúpula (Figura 4) se encuentra actualmente ubicada en la sala 26 del Museo Arqueológico Nacional, con $\mathrm{n}^{0}$ de registro 63.585 , y en la ficha correspondiente (20) se la describe como "Techumbre octogonal (sic) del tipo cupular ataujerado", indicando en la clasificación que "Su estructura es similar a otra en el palacio de Tordesillas. Balbina Martínez Caviró la clasifica dentro del grupo de armaduras semiesféricas ataujeradas, de forma cupular, del mismo estilo que la del Salón de Embajadores del Alcázar de Sevilla y la de la escalera principal de la Casa de Pilatos, esta última de comienzos del siglo XVI”. Quizá la adscripción de esta armadura como del tipo "ataujerada" podría venir de la referencia del trabajo de Martínez Caviró (21), quien categóricamente manifiesta que "son necesariamente ataujeradas las armaduras de cinco o siete paños, las cupulares y las de medio cañón” (p. 249). Como veremos, se trata de una armadura apeinazada que, como en todo trazado incluye algunos elementos auxiliares de lazo sin sección completa, lo que no indica que por ello se pueda definir como ataujerada.

También cabe reseñar que denominar como "octogonal" la cúpula de Torrijos es un error al que seguramente induce la configuración del tambor sobre el que se apoya, el cual tiene esa forma debido a los dos niveles de estribos y cuadrales paralelos entre sí. No obstante, es la media naranja la que conforma la auténtica techumbre, y su condición de semiesfera

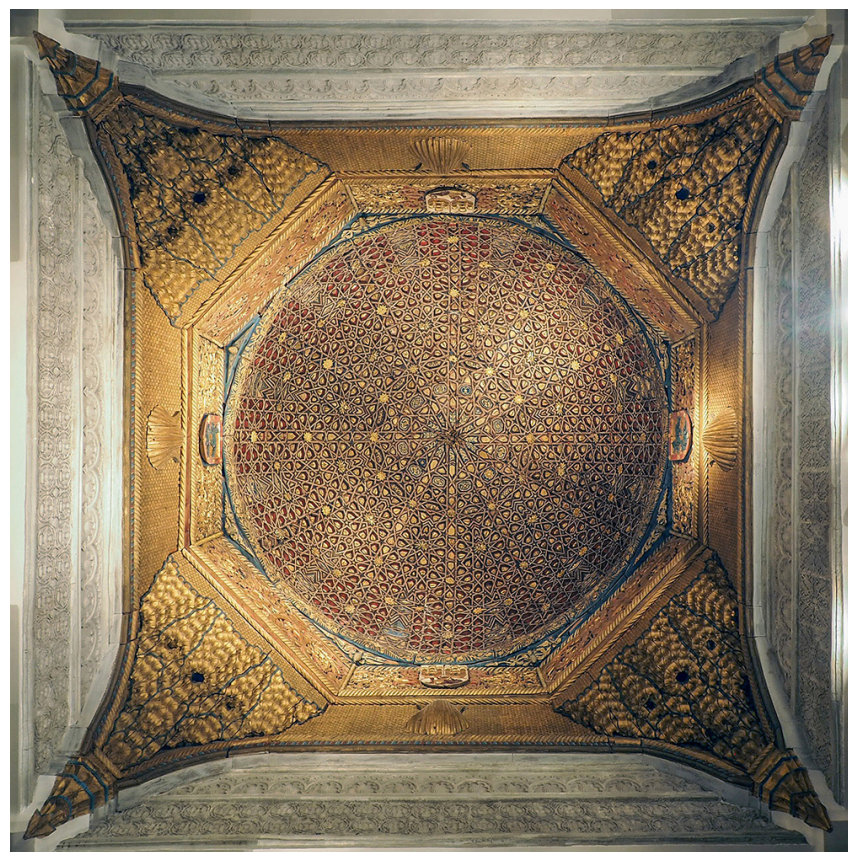

Figura 4. Vista del intradós de la cúpula de Torrijos. debe prevalecer sobre cualquier otro elemento a la hora de definir el conjunto, puesto que además, al referir una armadura como octogonal, crea confusión al poder tratarse de un ejemplar ochavado.

Asimismo, no es correcto relacionar la cúpula con la armadura ochavada de la capilla mayor del desaparecido Palacio Real de Tordesillas, puesto que se trata de una armadura de cinco paños ochavada por un extremo, cuya estructura y técnicas de trazado y ejecución, difieren de las de una media naranja, aunque su aspecto externo induzca a pensar lo contrario.

La sala del museo donde se ubica la cúpula reproduce con notable perfección las dimensiones originales que debió tener el salón correspondiente del palacio de los Cárdenas. En la primera planta del museo, sobre esta sala, existe una habitación de mayor tamaño cuyo suelo se ubica justamente por encima del nivel de las pechinas de mocárabes, lo que permite la visión y el mantenimiento del trasdós de la cúpula. Sería de enorme interés, y desde aquí se propone a la dirección del museo, hacer visible tal construcción por su cara oculta, mediante el acristalamiento de alguno de los paramentos de la estancia que precisamente lindan con una sala del museo.

\subsection{Descripción física}

La cúpula tiene un diámetro ecuatorial de $520 \mathrm{~cm}$. aproximadamente, con una sección de arco rebajado verticalmente unos $50 \mathrm{~cm}$., por lo que en realidad su forma es elipsoidal, con sus ejes mayor y menor dispuestos de manera horizontal y vertical respectivamente, pero con una escasa diferencia relativa entre ambos.

Obtenemos tal sección elipsoidal como resultado del análisis del trazado de lazo que después veremos en apartado 6 . Efectivamente, para conseguir una correcta concatenación del lazo el carpintero, muy seguramente, se vio obligado a desviarse de la geometría esférica pura.

A su vez, la cúpula presenta un bolsor bajo el plano ecuatorial de unos $20 \mathrm{~cm}$. La estancia que la contiene actualmente en el museo, así como la que hizo lo propio en el palacio torrijano, tiene una planta cuadrada de unos $550 \mathrm{~cm}$. de lado, lo que se ajustaba a 20 pies castellanos de luz a salvar entre muros.

La transición entre el círculo de base de la cúpula y el cuadrado de la planta, se produce mediante dos niveles de estribos que generan un prisma octogonal intermedio acabado por grandes tablas de alicer, y bajo las que son oblicuas, se sitúan las pechinas de mocárabes. A su vez, sobre el nivel superior del tambor octogonal existen unas delgadas pechinas de ajuste, de tamaño muy inferior a las primeras, que son las encargadas de realizar el paso entre el octógono y el círculo.

En cuanto a la cúpula propiamente dicha, presenta un trazado de lazo lefe en toda su superficie, compuesto exclusivamente por ruedas de 10 brazos. No obstante, al tener que adaptar un trazado originalmente plano a una forma cuasi-esférica, no se conservan sus propiedades geométricas y es necesario recurrir, a lo largo de determinados ejes meridianos, a la ruptura de dicho trazado, adaptándolo de manera arbitraria pero repetitiva en cada uno de los 12 gajos que componen la cúpula. De esta manera, los gajos, delimitados por camones principales, vienen a confluir en la clave, formando una estrella de 12 puntas. 


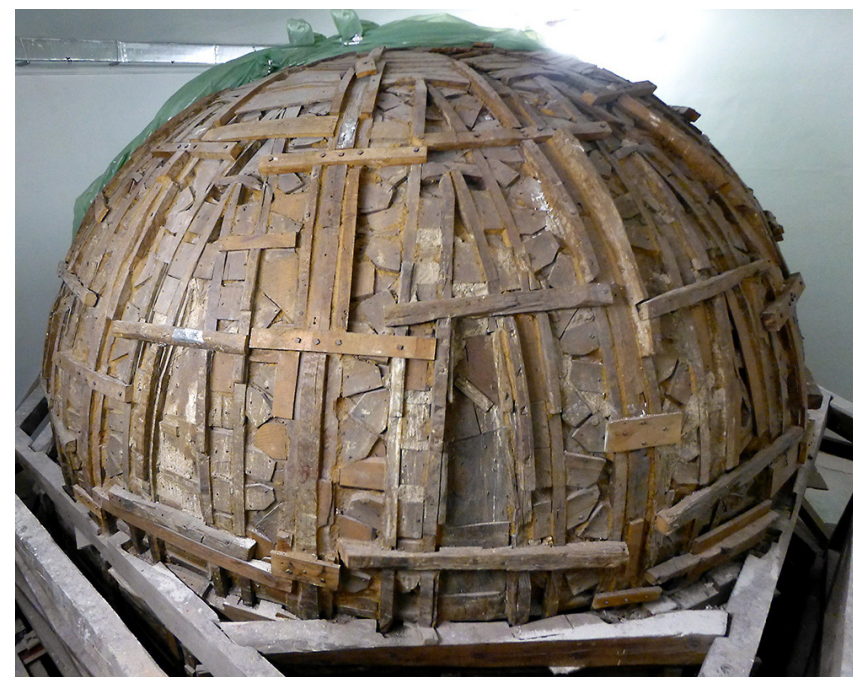

Figura 5. Trasdós de la cúpula de Torrijos, sobre su tambor original octogonal.

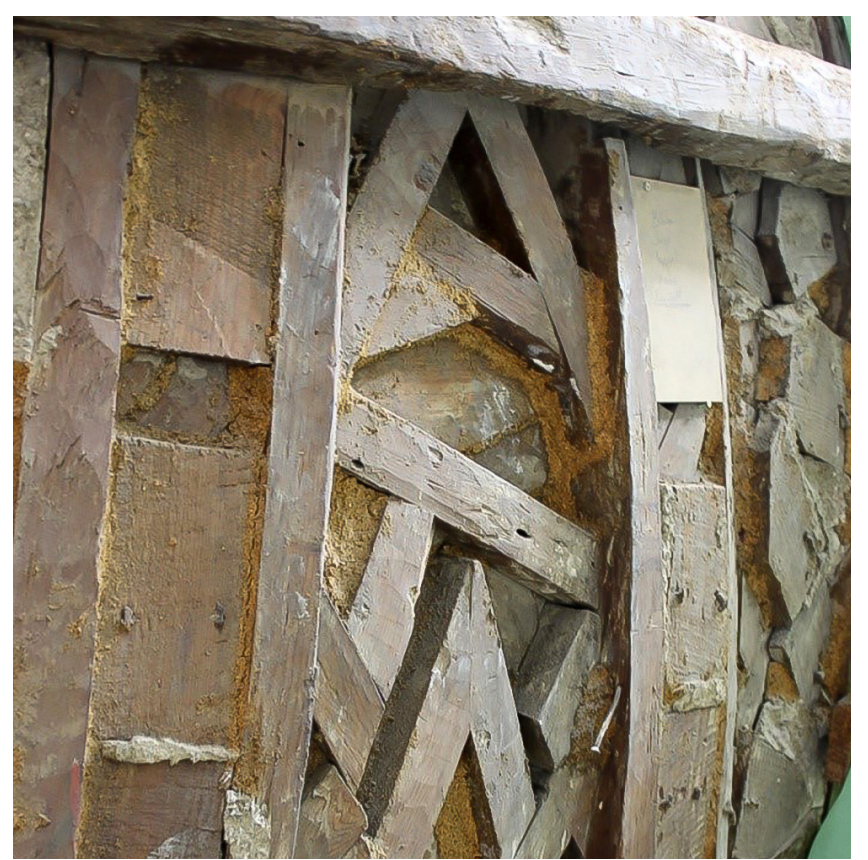

Figura 6. Detalle de del apeinazado de los paños, visible por el trasdós.

Cabe describir la presente techumbre como una armadura apeinazada con superficies parcialmente ataujeradas, en la que la mayoría de los elementos que la configuran tienen sección completa y plena función estructural, lo cual puede apreciarse en las figuras 5 y 6 , donde diversos elementos secundarios de la lacería son en realidad peinazos, y los espacios intersticiales se plementan con tabla.

\section{LA CONSTRUCCIÓN DE LA CÚPULA}

\subsection{Estribado}

El estribado, al que desafortunadamente algunos autores aún denominan durmiente, aunque oculto, es una parte fundamental de la armadura, puesto que tiene la misión de recibir el apoyo de los pares que conforman la cúpula, transmitir su carga a los muros perimetrales y a su vez soportar los empujes radiales que le acometen. Por otro lado, a nivel formal, es donde se produce la transición entre el círculo, el octógono y el cuadrado.

Se conserva en buen estado gran parte del estribado original, si bien aparecen algunos elementos añadidos. Está formado por dos niveles diferenciados: el inferior sobre la cota que determinan las pechinas de mocárabes, y el superior en la base de la cúpula, siendo la distancia libre entre ellos de unos 80 $\mathrm{cm}$, que se corresponde aproximadamente con la anchura de las tablas de alicer que sirven de acabado interior del tambor (Figura 7).

El nivel superior del estribado está configurado en forma de doble anillo, con piezas de madera de $20 \times 15 \mathrm{~cm}$ de sección, de manera que el anillo interior es el que recibe los camones sobre la cara superior de sus piezas, formando un conjunto hexadecagonal ensamblado a propósito para transmitir tanto las cargas verticales como los empujes horizontales al anillo exterior. Para el adecuado traslado de cargas, los maderos de ambos anillos se ensamblan a medias maderas que impiden el desbaratamiento del conjunto por tracción (Figura 8).

Una vez que el doble anillo superior del estribado recibe las cargas, las transmite al nivel inferior, formado por un único anillo, al cual se une mediante un conjunto de montantes verticales y cruces de san Andrés que tienen indicios de ser de factura reciente. Posiblemente en su día tuviese montantes y jabalcones en forma de $\mathrm{V}$ invertida tal como ahora representamos, de escuadría semejante a la de los estribos, de manera que las cargas del conjunto fuesen a concentrarse ya

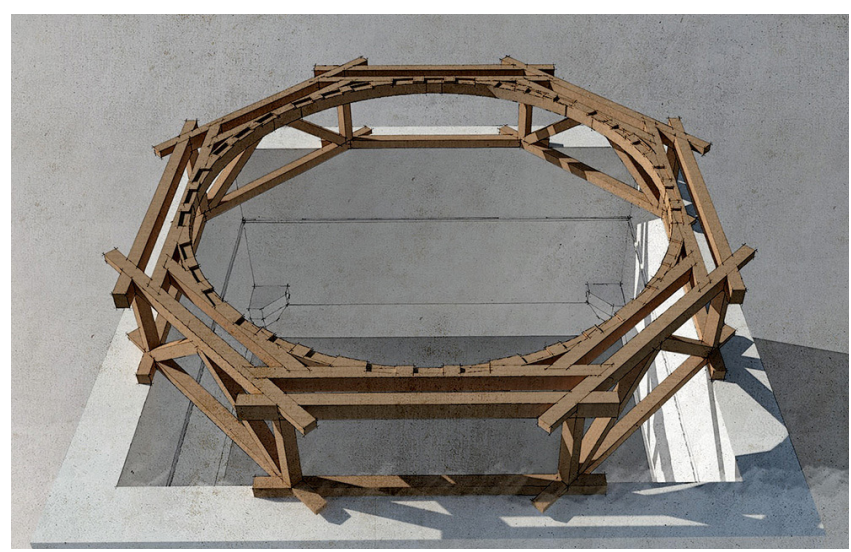

Figura 7. Estribado de la cúpula, sobre las cabezas de los muros de la estancia.

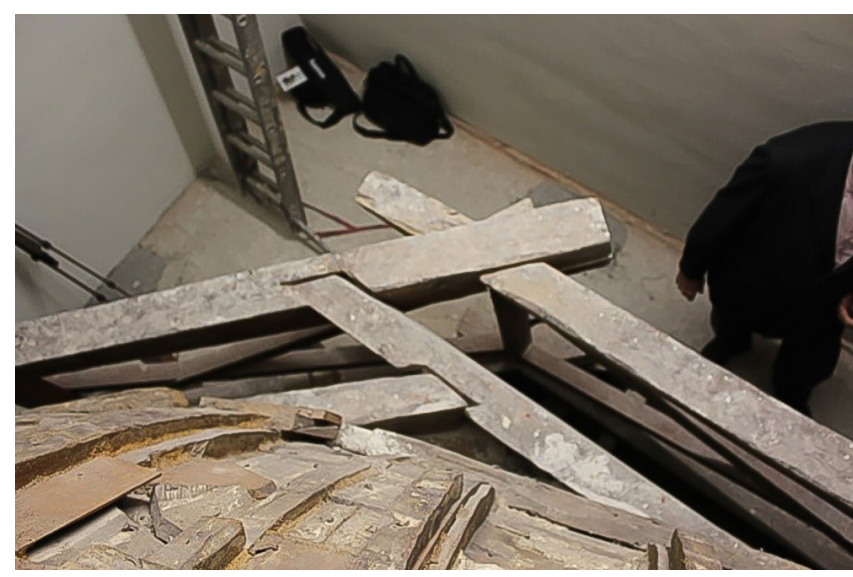

Figura 8. Detalle de unión del estribado de la cúpula. 
sobre los muros, dejando que los cuadrales del nivel inferior tuvieran como misión principal el soporte de las pechinas de mocárabes, y a su vez los estribos de ese mismo nivel una función principalmente de elemento de transición con el muro, a modo de solera.

\subsection{Estructura resistente y proceso constructivo de la cúpula}

En la construcción de esta cúpula se plantean dos principales incognitas, por una parte, cuál es su estructura portante y su secuencia constructiva y por otra, cómo el carpintero fue capaz de incorporar en ella la geometría de lazo.

Uno de los descubrimientos más importantes de la carpintería de armar española fue la duplicación de las limas, denominándose limas mohamares a las que cumplen tal condición, en sustitución de la lima simple o bordón, ubicada en la arista que conforman dos faldones adyacentes. La duplicación de la lima permite que cada paño de la armadura tenga autonomía estructural y posibilita la construcción en el suelo de grandes fragmentos que posteriormente pueden ser izados y montados con gran precisión. Se trata quizá de uno de los primeros ejemplos de prefabricación en la historia de la construcción.

Hemos podido verificar que en la construcción de esta cúpula los carpinteros supieron aplicar tal conocimiento y se dio un nuevo paso utilizando la calle de limas moamares, usualmente vacía de todo elemento estructural, para incorporar en ella lo que podría denominarse camones estructurales, que quedan ocultos, y a partir de ahí incorporar el resto de los elementos. Así, la cúpula puede entenderse como un sistema de pares, que sigue las reglas generales de la carpintería de lo blanco, formada por doce gajos independientes en forma de sector cuasi-esférico (Figura 9).

La estructura resistente de la cúpula está constituida por doce camones principales de unos $9 \times 7 \mathrm{~cm}$ de sección que apoyan en el estribado, funcionando éstos a nivel mecánico como "limas" o "nervaduras" entre los que se ubican, a modo de plementería resistente los diferentes gajos. En la zona de la clave, cuyo punto central estas piezas no llegan a alcanzar, un anillo de hierro forjado concéntrico con el eje vertical une las cabezas de todos estos maderos entre sí.

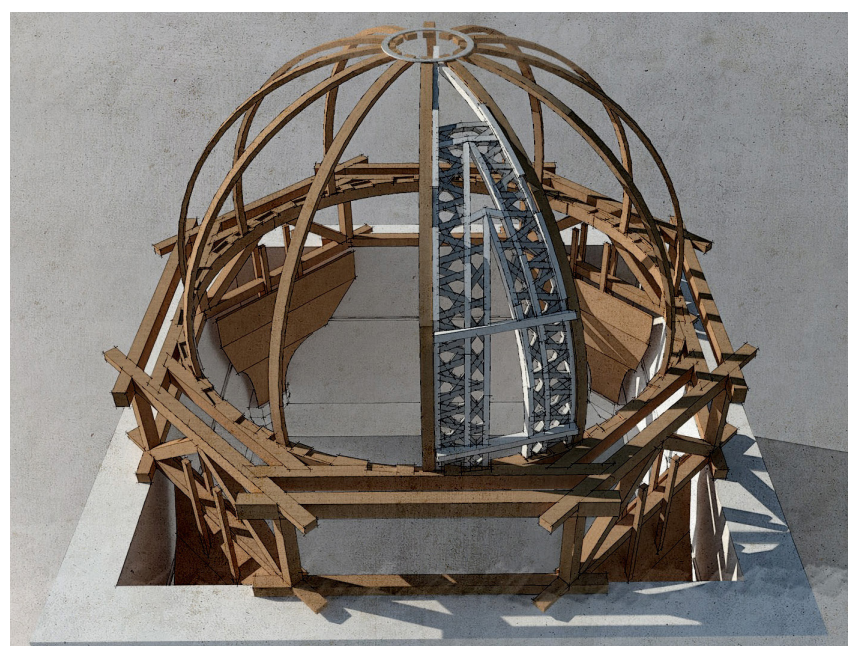

Figura 9. Estructura principal de camones unidos por el anillo metálico para la colocación posterior de los gajos.
Por su escuadrado con azuela y su idéntico aspecto al resto de las piezas, es muy probable que esta estructura sea la original, y se ejecutaría en primer lugar, para servir de armazón autoportante sobre el que ir asentando los gajos que se habrían prefabricados en el suelo. Si bien la idea de una subestructura sobre la que ir armando toda la cúpula es lógica, surge un problema de coherencia constructiva en el entorno de la clave, en el que actualmente se pueden apreciar distorsiones que impiden la continuidad del lazo de la rueda central de doce brazos. Es probable que la falta de continuidad material en la clave, unido al comportamiento reológico de la madera, hayan distorsionado el conjunto, tras sucesivos procesos de montaje, produciéndose una rotación del anillo y de las piezas que le acometen (Figura 10).

Cada paño, en forma de huso esférico, está conformado por dos familias de tres camones cada una, con una sección recta de 5,5 por $9 \mathrm{~cm}$ aproximadamente. Dentro de la misma familia, los camones tienen generatriz en forma de arcos de igual radio, contenidos en planos paralelos. Los principales y más largos de todos son los que conforman los límites meridianos del sector, y son paralelos y adyacentes a los camones principales de la estructura. El resto de los camones conserva el paralelismo, mientras que su longitud está delimitada por su encuentro con el camón correspondiente de la otra familia. Las distancias entre camones están predeterminadas por la inclusión del lazo de diez, lo que provoca la secuenciación irregular entre ellos, dejando el sector central comprendido entre los camones más cortos de cada familia como un área de ajuste, en el que debido a la colisión de trazados no es posible mantener su ortodoxia.

Singularmente, los camones principales quedan retranqueados con respecto al plano curvo en que se ubican los camones laterales de los gajos y de esta forma pasa visualmente inadvertida desde el intradós la presencia del camón principal, y puede producirse la continuidad de taujeles que forman la laceria cuyos sinos precisamente coinciden con la calle donde se ubican estos camones como podemos apreciar en el croquis de una sección radial (Figura 11).

Por otra parte, ninguno de los camones es contínuo en toda su longitud. La existencia de piezas de refuerzo de los empalmes, unidas a los camones por su trasdós mediante

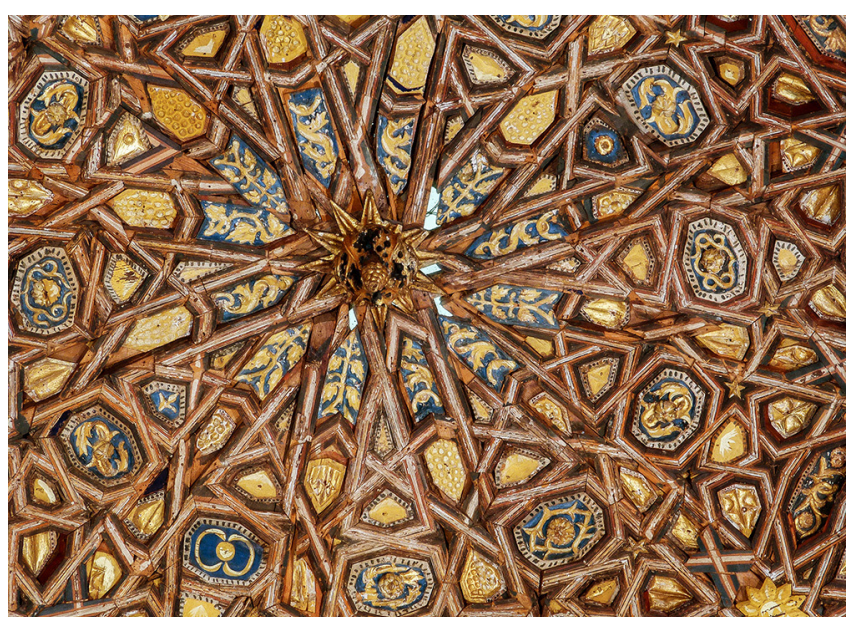

Figura 10. Zona de la clave de la cúpula vista por el intradós, con severas imperfecciones causadas por la discontinuidad de los camones. 


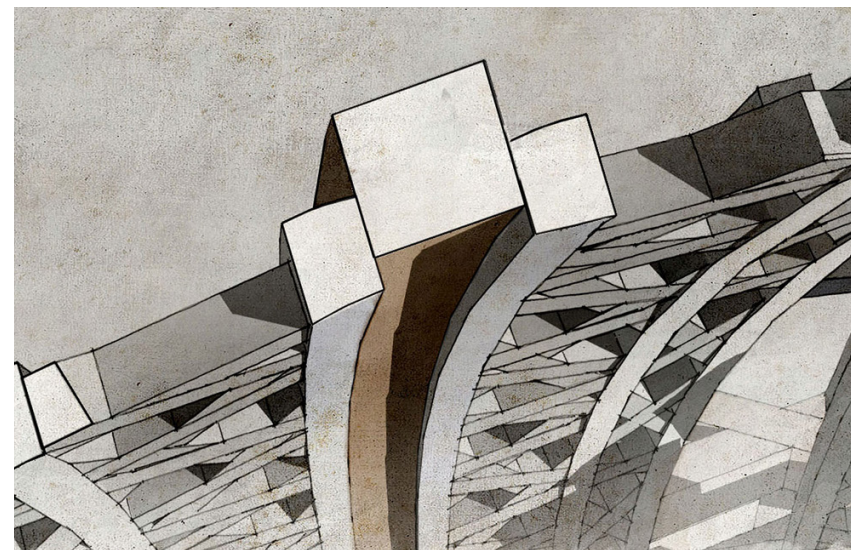

Figura 11. Sección radial de la cúpula, apreciándose la posición del camón principal y camones laterales de gajos adyacentes.

tornillos barraqueros, dan la pista de cuántas piezas componen cada camón. En el caso de los principales se dividen en tres partes, y en los camones de segundo y tercer orden son dos.

El proceso constructivo que se pudo seguir fue construir en primer lugar los doce camones referidos que forman el esqueleto básico de la cúpula, y tras encajarlos equidistantes en el estribo, unirlos en la parte superior con el anillo de hierro forjado, con lo que quedaría formada una estructura que va a soportar los diferentes gajos de la media naranja. Para la construcción de cada gajo es presumible que se hicieran un par de camones más para facilitar la prefabricación de cada gajo, de esa forma se podrían disponer en el suelo, en un bastidor a medida, separados una distancia igual a la que resultó en la estructura antes mencionada (Figura 12). Una vez fabricados todos los gajos, el siguiente paso sería el izado y colocación de los mismos, encajándolos en la estructura de base (Figura 13), y apoyándolos sobre el estribado mediante "caja y espiga”, tras lo cual, únicamente restaría cuajar el lazo de las calles que los separan. En condiciones ideales, hubiese sido posible incluso prefabricar los peinazos y taujeles necesarios para completar dicha calle, que en teoría es la menos distorsionada geométricamente. Sin embargo, dos traslados a lo largo de su historia, han debido provocar deformaciones considerables en su anchura y trazado. Ignoramos si en el primer montaje de la cúpula ya existían imperfecciones semejantes, pero a la vista de la actual recomposición, seguramente

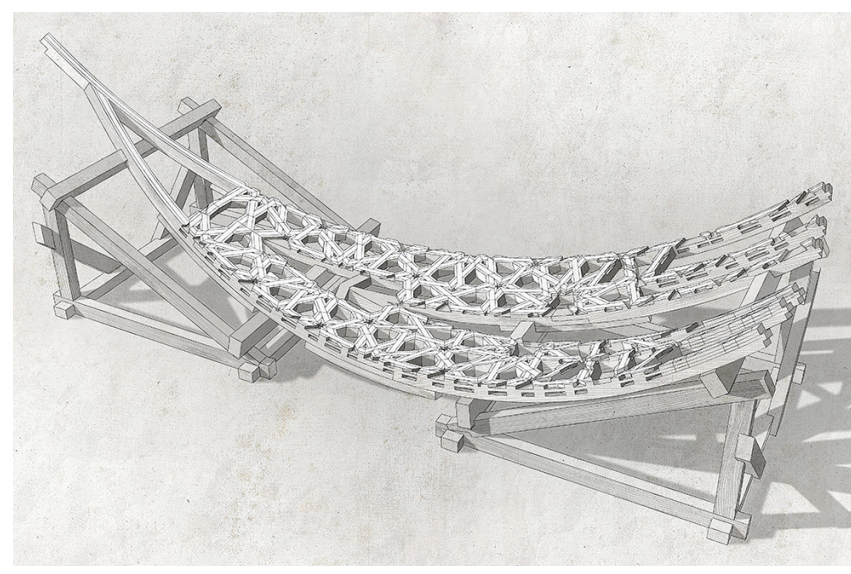

Figura 12. Hipótesis del montaje de un gajo sobre un bastidor a medida.

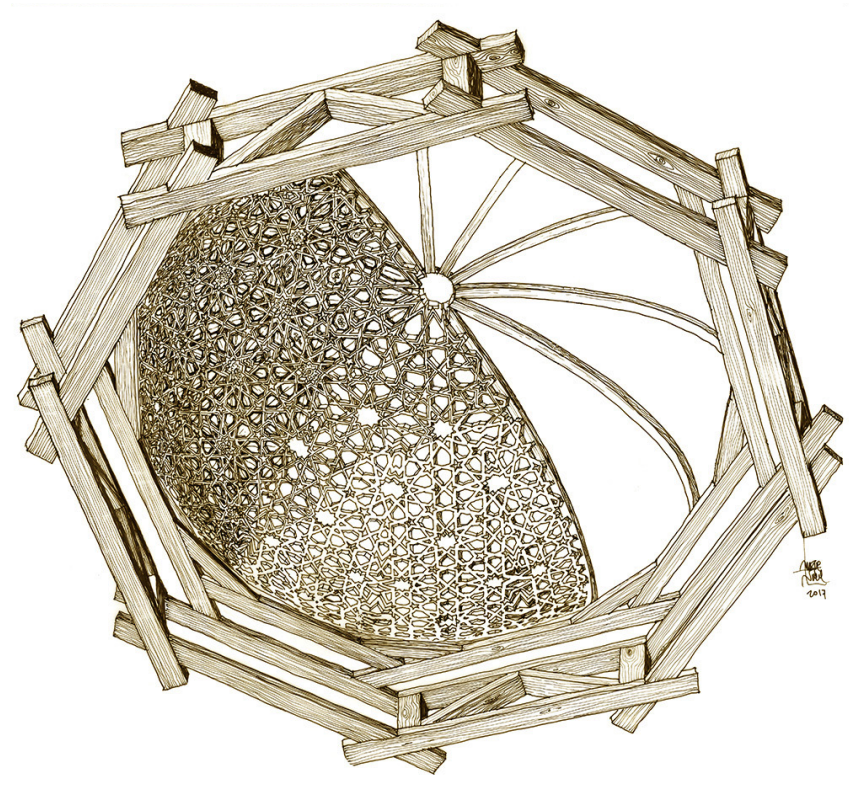

Figura 13. Vista inferior de la cúpula, en diferentes fases del proceso de montaje de los gajos sobre los camones principales.

era imprescindible un replanteo in situ de las piezas de las calles principales, para lo cual tuvo que existir un andamio bajo la cúpula en el que poder trazar y cortar con relativa comodidad.

A diferencia de la cúpula torrijana, en la de los Reales Alcázares de Sevilla, la circunstancia de la acumulación de distorsiones en la clave se resolvió con singular maestría, puesto que según Candelas-Gutiérrez (14), el casquete esférico de la zona superior se realizó de manera unitaria, y tras su colocación se fueron añadiendo el resto de los paños a su alrededor, de manera que los posibles errores se distribuían entre todos los husos.

\section{TRAZADO DE LAZO: GEOMETRÍA Y EJECUCIÓN}

A día de hoy, en las pocas medias naranjas que existen, se desconoce por completo cómo sus autores introdujeron el trazado en ellas lo que, en el presente caso de Torrijos, nos obliga a movernos en el terreno de las hipótesis. Lógicamente, hoy sabemos cómo trazar con precisión los límites del gajo correspondiente a un doceavo de una esfera, por lo que nada más sencillo que hacer dicha plantilla para ir dibujando en ella la lacería que podemos ver en la cúpula real. Sin embargo, al primer intento surgen los problemas: la parte baja del gajo tiene que ser mucho más ancha en relación con las ruedas, ya que no cabe toda la traza que vemos en la obra original, mientras por la parte alta los espacios intermedios de la cúpula son menores que en la traza realizada.

Eso quiere decir que el huso de la media naranja, dividida en doce gajos, no puede inscribirse en una superficie esférica, lo que complica enormemente averiguar cuál fue la forma real, original, de la cúpula. Para tratar de conseguirlo hay que proceder por tanteos sucesivos, ensanchando el huso por la parte baja hasta que el trazado de lacería se asemeje lo más posible al real. Eso dará lugar a que la teórica clave de la cúpula se encuentre por debajo de la que sería esférica, pero en cada intento realizado es imprescindible corregir los bordes del huso resultante. Al final fueron necesarias doce aproximaciones 
para llegar a una solución bastante parecida a la realidad, y donde se aprecia que la sección de la cúpula resultante está muy por debajo de la que correspondería si fuera hemisférica (Figura 14).

Si llegar a esta solución con los medios que hoy disponemos, ha resultado tan complicada, ¿̇cómo pudo su autor construirla sin posibilidad de cometer errores?. La única posibilidad imaginable es que no le preocupaba en absoluto que fuera esférica, las explicaciones de López de Arenas (22) son sin duda las de alguien que nunca hizo una media naranja, ya en 1619 el propio López de Arenas advertía sobre la creciente pérdida de tal bagaje constructivo.

Con respecto a la incorporación del trazado de lazo de diez en los gajos, es probable que se comenzase cada familia de camones de manera independiente. Se partiría de los camones principales, en los que es más fácil mantener el control geométrico, puesto que al hallarse junto al límite de los paños y paralelos al mismo, la distorsión angular que se produce es menor. Dicho límite es el eje sobre el que se ubican los centros de las estrellas de diez puntas, de donde parten los trazos que se dan con los cartabones de cinco y diez, y que sirven a su vez para realizar las ruedas, y para posicionar unas respecto a otras (Figura 15).

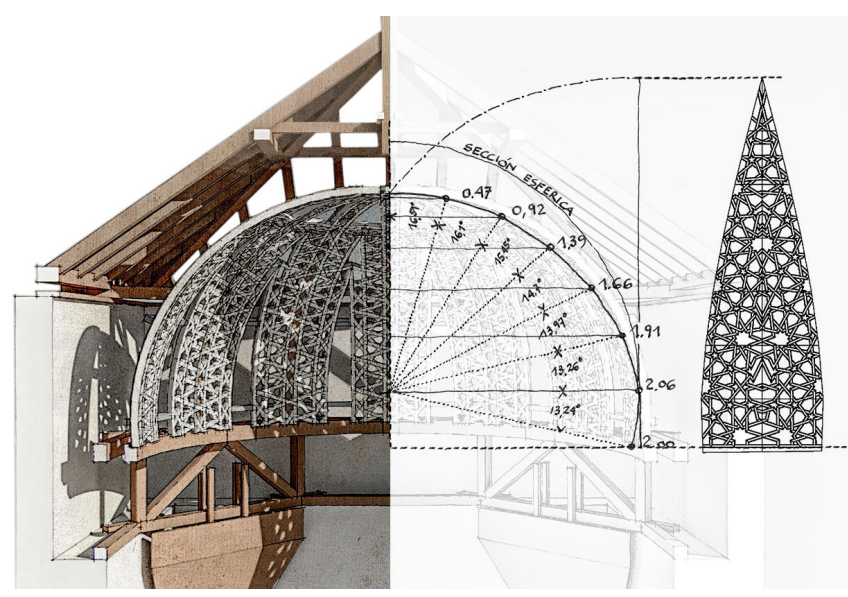

Figura 14. Representación de la sección elipsoidal de la cúpula basada en el trazado geométrico definitivo de la lacería presente en el gajo.
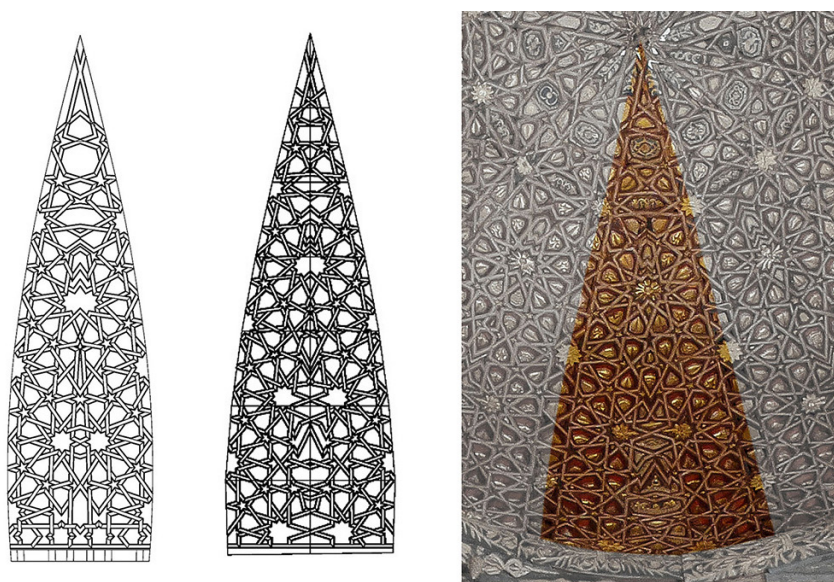

Figura 15. Aproximaciones gráficas al trazado de un gajo (izquierda y centro), y fotografía del intradós en la que se destaca en color un gajo (derecha).
Cada camón va a representar la calle de las medias ruedas que forman cada borde, cuyos diámetros medirán aproximadamente una sexta parte de la altura del huso. Una vez dispuestas las medias ruedas de los bordes, se colocarían las ruedas tangentes en una calle paralela, dispuesta lógicamente hacia el centro, cuidando de no rebasar el eje vertical del huso, y sobre la marcha se va rellenando el espacio central según el mejor criterio del propio tracista, y una vez completado un huso y afianzadas las diferentes piezas entre sí, se procedería a izarlo, sujetándolo ajustado entre dos camones, repitiendo el proceso hasta completar los doce gajos (Figura 16). Tras terminar estos pasos sólo falta colocar los taujeles que completan la lacería de las calles entre las medias ruedas existentes a cada lado del camón estructural.

El posicionamiento de las estrellas en lo que sería la "calle de limas”, iría en paralelo al de las mismas en la tercera calle, teniendo cada familia de camones un trazado prácticamente ortodoxo salvo por las imperceptibles correcciones que habría que hacer para adaptarlo a la superficie esférica. Sin embargo, en una fase posterior, a la hora de unir ambas familias mediante la zona de ajuste central, el trazado resultante a lo largo de la unión sería una solución coyuntural en la que el carpintero intentaría que los apaños pasasen lo más desapercibidos posible.

La unión de ambas mitades de cada gajo, se vería reforzada por diversas piezas colocadas en dirección horizontal, dos cortas en los vértices ubicados en los encuentros de camones, y otras dos más largas hacia la mitad inferior del paño, abarcando todos los camones del huso con la intención de estabilizarlos. Estas piezas solo se pueden observar por el trasdós de la cúpula.

Finalmente, en aras de una unión efectiva entre gajos y estructura, se añadieron maderos horizontales que mediante tornillos barraqueros afianzan toda la armadura, pasando por detrás de los camones estructurales. Por su aspecto y configuración se deduce que son posteriores a la fabricación de la cúpula, lo cual no excluye la posibilidad de que en el primer montaje existieran otros que cumplieran idéntica función.

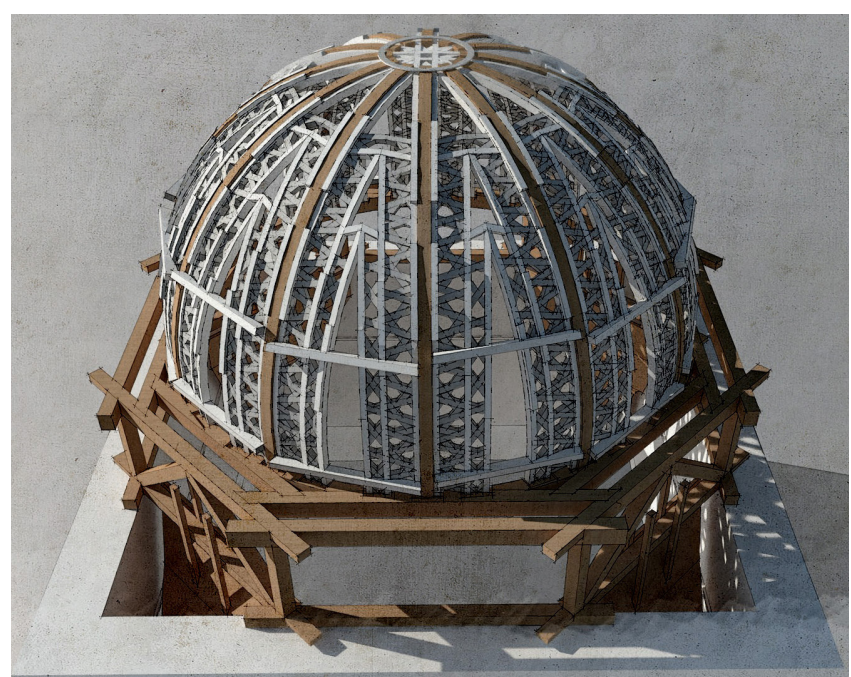

Figura 16. Representación del extradós de la cúpula con todos los gajos, excluyendo el entablado para apreciar la estructura apeinazada. 


\section{ELEMENTOS AUXILIARES}

\subsection{Alicer y zona de transición}

En la parte más baja de lo que sería el alicer, se colocan una serie de tablas con decoración ajedrezada sobre la que se sitúan grandes vieiras de peregrino. Esta franja, a pesar de ser de madera, está sobrepuesta al muro y no tiene una subestructura que la soporte, salvo una moldura inferior de cordón que hace las veces de solera. Se ubica en el mismo nivel que las pechinas de mocárabes, por lo que únicamente se coloca en los cuatro lados coincidentes con muros.

Un nivel por encima, las tablas que dan forma y sirven de terminación al tambor octogonal sobre el que se asienta la cúpula (Figura 17), se construyen de modo que se encuentran contrapeadas y unidas entre sí por largueros en su trasdós. Discurren por delante de la cara interna de los estribos, ocultándolos y generando ocho planos sobre los que la decoración vegetal y heráldica se ve rematada en cada arista por molduras de cordón. Los anillos superior e inferior de estribos y cuadrales son los que sirven de soporte de dicho entablamento, y en el caso del anillo superior interno, una serie de cuadralillos, que efectúan la transición del octógono al círculo, son los que permiten el apoyo de la cúpula en zonas en las que de otra manera sus camones quedarían en el aire, puesto que el círculo de base de la media naranja tiene un diámetro inferior a la luz de la estancia.

Como banda de transición ornamental, se colocan una serie de tablas curvas sobre los mencionados cuadralillos, actuando a modo de almarbate, y ocultando tras de sí el arranque de los camones sobre el estribamento.

\subsection{Pechinas de mocárabes}

Las pechinas de mocárabes, compuestas por distintas adarajas de cuerpo prismático, penden en este caso de cuatro nabos cada una, los cuales están atravesados en su zona superior por dos barras metálicas que unen cada pareja de nabos y les da apoyo sobre el cuadral inferior del estribado (Figura 18). No obstante, como refuerzo para no depender exclusivamente de elementos que trabajan a tracción, el extremo inferior de las pechinas apoya sobre una suerte de zapata de fábrica anclada al muro, de manera que entre ambos elementos confieren estabilidad a la pechina.

\section{CONCLUSIONES}

La cúpula de lazo lefe del Palacio de los Cárdenas supone un documento construido de uno de los tipos de armadura cuya realización se consideraba patrimonio exclusivo de los "geométricos", esto es, del escalafón más alto de los carpinteros de lo blanco. Si bien su trazado se asemeja en gran medida al de las otras tres cúpulas que han sobrevivido el paso del tiempo, sus peculiaridades constructivas, tales como la subdivisión de los gajos o la subestructura, muy diferentes a las cúpulas sevillanas, por ejemplo, nos permiten entrever que

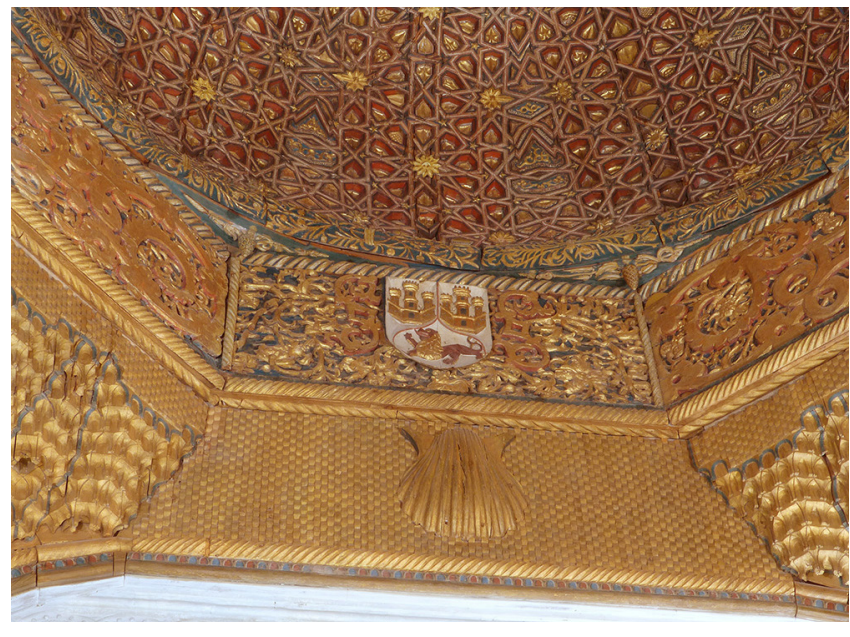

Figura 17. Vista general de las tablas de alicer, cubriendo el tambor octogonal con decoración heráldica y floral, y la banda de transición del octógono al círculo.
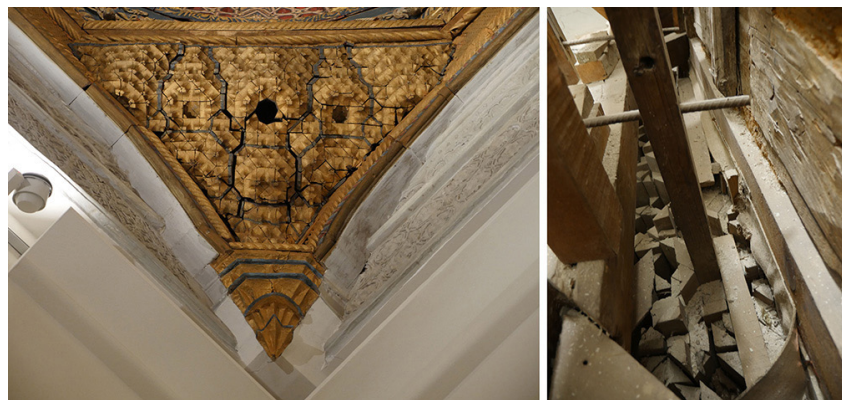

Figura 18. Pechinas de mocárabes pendiendo del estribado inferior. Vista inferior de los mocárabes (izquierda) y nabo anclado al estribo (derecha).

no existía un único camino para llegar a la media naranja, y que era potestad del carpintero idear y ejecutar la estructura y su montaje.

Adicionalmente, es patente una vez más, que en la carpintería de lazo, el afán de perfección geométrica queda supeditado a un equilibrio entre la estética y la simplicidad técnica. Así, encontramos que no se consideraba prioritario que el proceso de trazado y ensamble estuviese dominado por una férrea aplicación de la geometría esférica, ya fuese tanto en su dimensión vertical como en la horizontal. Es particularmente interesante que la elaboración de los gajos tuviese como base la aplicación de la lacería de diez casi como de un elemento plano se tratase, con sus mismos cartabones y escopladuras, sobre los camones curvados con un radio determinado, tras lo cual, al unir dos familias de camones simétricos, quedaba conformado el gajo, cuyas dimensiones exteriores se habían establecido previamente. Podemos aseverar que del estudio directo de las cúpulas de lazo, tal y como es el caso presente, se pueden recomponer saberes técnicos que ni los propios tratadistas pudieron o supieron incluir en sus obras, lo cual supone un importante avance en el conocimiento de la carpintería de lo blanco. 


\section{REFERENCIAS}

(1) Pavón Maldonado, B. (2010). Bóvedas y cúpulas en la arquitectura árabe de occidente. Inventario y reivindicación. Obtenido de http://www.basiliopavonmaldonado.es/Documentos/Cupulas.pdf

(2) Hurtado Valdez, P. (2012). Bóvedas Encamonadas: Origen, Evolución, Geometría y Construcción entre los Siglos XVII y XVIII en el Virreinato de Perú. Tesis doctoral. Madrid. UPM.

(3) Prieto y Vives, A. (1932). La carpintería hispano-musulmana. Arquitectura: órgano de la Sociedad Central de Arquitectos, 161-162: 265-302.

(4) Nuere Matauco, E. (2008). La carpintería de armar española. Madrid: Munilla-Lería, 333.

(5) González de León, F. (1844). Noticia artística, histórica y curiosa de todos los edificios públicos, sagrados y profanos de esta muy noble ciudad de Sevilla. Sevilla: Imprenta de José Hidalgo.

(6) Fernández Aguilera, S., \& Pérez Ferrer, J. (2000). Restauración de la cúpula del Salón de Embajadores. Apuntes del Alcázar, 1: 74-85.

(7) Albendea Ruz, María Esther (2011). La Carpintería de lo Blanco de la Casa de Pilatos de Sevilla. Tesis doctoral. Universidad de Sevilla.

(8) San Cristóbal Sebastián, A. (2006). Nueva visión de San Francisco de Lima. Lima: Institut français d'études andines, 113-121.

(9) López de Arenas, D. (1633). Breve compendio de la carpintería de lo blanco y tratado de alarifes. Sevilla: Luis Estupiñán.

(10) San Miguel, F. A. (1640). Manuscrito. Conservado en la Universidad de Texas en Austin.

(11) Nuere Matauco, E. (1985). La carpintería de lo blanco. Lectura dibujada del primer manuscrito de López de Arenas. Madrid: Ministerio de Cultura.

(12) Toajas Roger, M. (1987). Carpintería de tradición mudéjar en la arquitectura española: Diego López de Arenas (Tesis doctoral). Madrid: Universidad Complutense de Madrid.

(13) Candelas-Gutiérrez, A. (2000). Bóvedas de madera: ¿se pueden construir según describen los tratados? En Actas del Tercer Congreso Nacional de Historia de la Construcción (págs. 193-204). Sevilla: Sociedad Española de Historia de la Construcción.

(14) Candelas-Gutiérrez, A. (2001). Geometría y proceso constructivo de la cúpula del Salón de Embajadores. Apuntes del Alcázar de Sevilla, 2: 5-25.

(15) Báez Macías, E. (1969). Obras de Fray Andrés de San Miguel. Ciudad de México: Universidad Nacional Autónoma de México.

(16) Nuere Matauco, E. (1980). La carpintería de lazo. Lectura dibujada del manuscrito de Fray Andrés de San Miguel. Málaga: Colegio Oficial de Arquitectos de Málaga.

(17) Repullés y Vargas, E. (1894). El palacio de Torrijos. Resumen de Arquitectura. Revista de la Sociedad Central de Arquitectos, 101-105.

(18) Pérez Suescun, F. (1998). La techumbre de Torrijos. Los palacios mudéjares. De la cueva al palacio: vivienda y vida cotidiana. En Museo Arqueológico Nacional, PIEZA DEL MES. Recuperado de http://www.man.es/dam/jcr:007b786a26d3-45c8-b6fc-dd2d6ddda122/man-pieza-mes-1998-12-techumbre-torrijos.pdf

(19) El palacio de Torrijos. (1902). Por esos mundos, 87. Abril: 342-347. Madrid. Recuperado de http://hemerotecadigital. bne.es/issue.vm?id=0003104933\&search=\&lang=es

(20) Museo Arqueológico Nacional. (s.f.). Techumbre del Palacio de Altamira. Ficha, Museo Arqueológico Nacional, Madrid. Número de inventario 63585. Recuperado de http://ceres.mcu.es

(21) Martínez Caviró, B. (1976). Carpintería Mudéjar Toledana. Cuadernos de la Alhambra, 12: 225-266.

(22) López de Arenas, D. (1633). Breve compendio de la carpintería de lo blanco y tratado de alarifes. Sevilla: Luis Estupiñan, 33V-34R. 